\title{
Increasing complexity can increase stability in a self-regulating ecosystem
}

\author{
James Dyke, Jamie McDonald-Gibson, Ezequiel Di Paolo, and Inman Harvey \\ Centre for Computational Neuroscience and Robotics \\ School of Science and Technology, University of Sussex, BN1 9QH, UK \\ jgd20@sussex.ac.uk, jamiemcdg@yahoo.co.uk, ezequiel@sussex.ac.uk, \\ inmanh@sussex.ac.uk
}

\begin{abstract}
A long standing debate within ecology is to what extent ecosystem complexity and stability are related. Landmark theoretical studies claimed that the more complex an ecosystem, the more unstable it is likely to be. Stability in an ecosystems context can be assessed in different ways. In this paper we measure stability in terms of a model ecosystem's ability to regulate environmental conditions. We show how increasing biodiversity in this model can result in the regulation of the environment over a wider range of external perturbations. This is achieved via changes to the ecosystem's resistance and resilience. This result crucially depends on the feedback that the organisms have on their environment.
\end{abstract}

\section{Introduction}

Prior to the 1970s, ecologists generally held the view that the more complex an ecosystem, the more stable it would tend to be [1][2]. These conclusions were based on the observation that terrestrial ecosystems that had undergone significant simplification (e.g. alien species invasion or human monoculture), were more prone to pronounced fluctuations in population density. This reasoning was challenged by the theoretical study of May [3] who, building on earlier work by Gardner \& Ashby [4], showed that the greater the number and strength of connections between species in a model ecosystem then the less stable it is likely to be. Notwithstanding the details of these particular theoretical studies, the relationship between complexity and stability has proved to be more subtle and elusive with the debate being conducted in two broad strands: a search for a general relationship between diversity and stability; investigating the relationship between food web structure and stability. See [5] for a review.

The stability of ecosystems can be assessed in terms of constancy, persistence, resistance, inertia and resilience. The results we will present will show that increasing ecosystem complexity reduces resistance (the ability to resist external pertubations), but increases resilience (the ability to recover after perturbations) in a population of two-locus, haploid individuals. These changes can result in an overall increase in the stability of the ecosystem as measured by its ability to regulate an environmental variable. Complexity in an ecosystems context can be 
defined and measured in a number of ways. For example increasing complexity may equate to increasing the number and type of ecological interactions. In the model we present, complexity is increased via increasing the rate of mutation that operates on both loci and thus increasing genetic diversity. It would be possible to vary the diversity of the population by means other than mutation. For example the influx of alien species could be modelled with the random replacement of individuals. However given the very simple individuals modelled this, with certain limitations, could be considered as equivalent to the mutation of existing individuals. The frequency of alleles within the population changes in response to changes in selective pressure that are produced by external perturbations that operate on an environmental resource which determines the fitness of all individuals. Crucially, the activity of the organisms also affects the environmental resource; there is a feedback loop from organisms to environment and back again.

\subsection{Self Regulating Models}

The model we present is a modified version of [6] initially formulated by McDonaldGibson which can be considered as a synthesis of the self-regulating Daisyworld [7] and niche construction models [8]. Organisms through their activity and metabolism will create, modify and at times destroy their environmental surroundings or 'niches', e.g. dams built by beavers, photosynthesis and the production of oxygen by plants or the burning of fossil fuels and production of carbon dioxide by humans. The theory of niche construction attempts to incorporate the feedback that organisms have on their environment into the established theories of population genetics and evolutionary theory [9].

Daisyworld features niche constructing-plants or 'daisies' that via their different colour affect the albedo of the planet they inhabit and thus the amount of energy it absorbs from the star it orbits. Both daisy types have an identical temperature-dependant growth function. This takes the form of an upside-down parabola that peaks at the optimum temperature for growth and declines on either side towards zero. Black daisies have a lower albedo than white daisies; therefore, in a cool environment, they absorb more sunlight, causing their local temperature to rise towards the optimal growth temperature. This increases their growth rate and engenders a feedback loop with their numbers rising, further increasing global temperature. This positive feedback on black daisy growth continues until the temperature moves towards the optimum for growth. The white daisies have a similarly regulatory effect and selective advantage in higher temperatures through cooling their environment. The net result is that the temperature on the planet is regulated around the optimum for daisy growth despite large increases in heat input from the star which increases in brightness over time. Since its inception it has been significantly extended with 1D, 2D and 3D implementations. See [10] for a review. The question of complexity and stability has previously been explored within the Daisyworld model [11][12][13]. These studies found that when feedback from organisms to the environment is incorporated in to their models, more complex and diverse ecosystems prove more 
robust to external perturbations. The McDonald-Gibson model exhibits regulation with fewer assumptions that these previous studies and is potentially more general. In particular, there are no pre-defined optimal resource conditions; the allele that determines which environmental conditions gives the greatest growth and fitness is subject to mutation and can take on a range of values, unlike the original Daisyworld model and variants listed above, and so regulation may occur over a wide range of 'optimal' environmental conditions.

\section{Methods}

A fixed population of two-locus haploid organisms is modelled. These interact with their environment by increasing or decreasing an environmental resource. This resource determines the fitness of the organisms. Real world resources may be temperature, $\mathrm{pH}$, concentration of a particular chemical in a well mixed solution or partial pressure of a gas. A proportion of the population reproduce by cloning offspring that are subject to mutation with a fixed probability. The same proportion of individuals die thus maintaining a constant population. Reproduction and death occur simultaneously at every unit of dimensionless time. This may represent an annual, daily or even hourly cycle. The ecosystem is perturbed over time by an external force. Real world forcing may be thermal driving operating on an entire biosphere from a star or increasing chemical input into a freshwater lake via runoff. In the absence of any organisms this linear forcing would result in a linear increase in the environmental resource. This ecosystem, whilst simple, features a feedback loop from environment to organisms to environment and so on. Therefore exact analysis is not trivial and an individual based model was produced to compute values for varying parameter values over a range of external perturbations. In this model the resource is represented by a single variable, $R \in[-50,150]$. The fitness $F$ of individuals is a function of $R$. Each individual has a genotype that specifies its effect on the resource and the resource level to which it is best adapted. The $\Theta$ locus specifies the phenotypic effect on the environmental resource. The $A$ locus specifies the resource level to which the phenotype is best adapted. The $\Theta$ locus has two possible alleles: $e$ which reduces the resource and $E$ which increases the resource. The bi-allelic $\Theta$ locus is represented with a double floating point number in the range $[-1,1]$. An individual will have the $e$ allele if $-1>\Theta<0$ and the $E$ allele if $0>\Theta<1$ The $A$ locus is represented with a double floating point number in the range $[15,85]$ and specifies the point that the individual is best adapted to within the range of resource values. Equation 1 is used to calculate an individual's fitness which is a parabolic function of the resource. This function peaks at the $A$ value and declines sharply on either side towards zero. The fitness of the $i$ th individual is a function of $R$ and $\lambda$ which provides a measure of the span of the parabola.

$$
F_{i}=\left\{\begin{array}{cc}
1-\lambda\left(A_{i}-R\right)^{2},\left|A_{i}-R\right|<\lambda^{-\frac{1}{2}} \\
0 & \text { otherwise }
\end{array}\right.
$$


Natural selection is modelled using a genetic algorithm with a proportion of the population subject to death, selection, and reproduction at each time step. A constant death rate, $\gamma$, is applied at each step. Therefore, on average, the lifetime of any individual is $1 / \gamma$ time steps. For each death, a tournament, where the victor is the individual with higher fitness, is held between two randomly selected members of the population. The winner of a tournament replaces the loser in the population. If both individuals fitness is the same, no replacement occurs. Mutation occurs with a probability of $\mu$ at each locus. This is performed by adding a number drawn from a Gaussian distribution of 0 mean and standard deviation 0.05 to the allele. The rate of change of the resource is:

$$
\frac{d R}{d t}=\alpha \sum_{i} \Theta_{i}+\beta(P-R)
$$

Where $\Theta_{i}$ is the effect that the $i$ th individual has on the resource value $R$. $P$ is the current amount of external perturbation that is being applied at time $t$. Constants $\alpha$ and $\beta$ determine the relative strengths of the population effects and external perturbation.

\section{Preliminary Results}

In the following results, unless otherwise stated, the maximum population size, $K$, is 2000 with the population being initialised with 2000 randomly generated individuals. The impact effect alleles $e$ and $E$ are set to -1 and 1 respectively. $\lambda$ is fixed at 0.04 for all individuals which gives a range of 10 resource values in which an individual will have non-zero fitness. Mutation rates, $\mu$, for alleles at both loci is fixed at 0.1 The death rate, $\gamma=0.01$, and consequently 20 individuals are selected for tournament reproduction at every time step with the winners replacing the losers within the population. Simulations consist of 100,000 discrete time steps. Simulations were first performed with no population effects operating on $R(\alpha=0)$. The external perturbing force, $P$, was increased linearly from 0 to 100 over the course of a simulation run. As $R$ increases, the population tracks this change with the average $A$ allele, $\bar{A}$, quickly converging to $R$ and increasing linearly. The population simply responds to the changing selective pressure produced by the external perturbing force. Simulations were then performed in which the the maximum difference, between the resource $R$, and perturbing force $P$, that can be maintained by the population is 50 $(\alpha=0.0025, \beta=0.1)$. Again, $P$ was increased linearly from 0 to 100 over the course of a simulation run. Initially $R$ increases linearly with $P$. However when the resource enters the range $[15,85]$ it no longer increases with increasing $P$, but stabilises at $\approx 15$. As $P$ increases, $R$ remains at the same value whereas the sum of population effects decreases. Results are plotted in Figure 1.

The model exhibits regulation in its resistance to the external perturbing force, $P$. This regulation proves to be robust to a wide range of parameter values. Regulation is still observed with the following changes (data not shown): population size can be reduced by a quarter (further reductions tend lead to 
unstable populations due to increased variance produced by the stochastic GA) and increased beyond 100,000 (available computation time considerations led to a population of 2000 individuals being used for data collection); the $\lambda$ parameter that determines the width of the fitness function can be halved or increased by a magnitude; the $\alpha$ and $\beta$ parameters can be increased to 0.05 and 1 and reduced to 0.00005 and 0.001 respectively; the death rate can be decreased by one and increased by two magnitudes; the discrete $e$ and $E$ alleles can be replaced with double floating point values over the range [-1,1]; the assumption of a fixed population can be replaced with a variable population with logistic growth dynamics up to a carrying capacity, $K$, the value of which can vary over the same range for the fixed population.

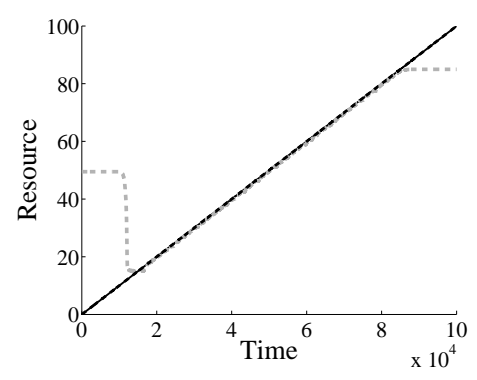

(a)

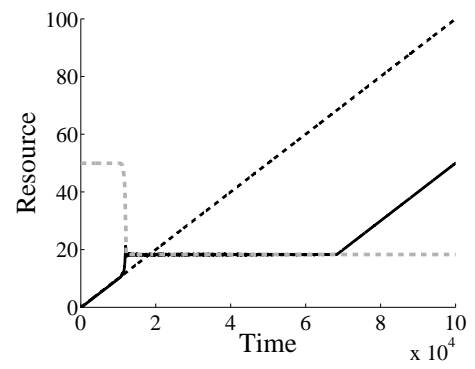

(c)

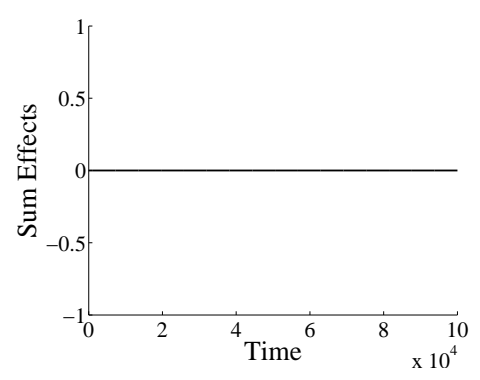

(b)

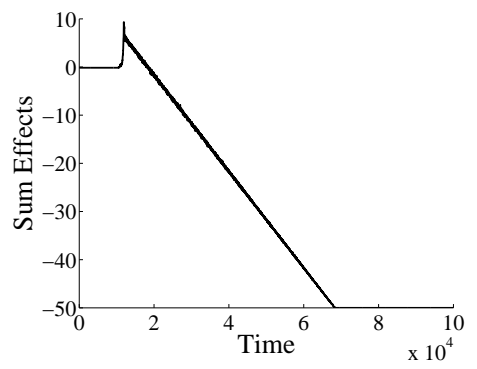

(d)

Fig. 1. Results of two simulations are plotted: no population effects on $R(\alpha=0)$ in (a) \& (b); with population effects on $R(\alpha=0.0025)$ in (c) \& (d). Time is plotted on the horizontal axis of all plots. The perturbing force, $P$, and the resource, $R$, are plotted with dashed black and solid black lines respectively in (a) \& (c) along with the mean $A$ trait of the population, $\bar{A}$, which is plotted with a dashed grey line. The effect the population has on the resource, $\sum_{i} \Theta_{i}$ is plotted in (b) \& (d). In both simulations $R$ initially increases with $P$ until $P$ drives $R$ into the range [15,85] at which point the population rapidly converges to a single $A$ trait: $\alpha=0, \bar{A} \approx 15 ; \alpha=0.0025$, $\bar{A} \approx 20$. With no population effects as $P$ continues to increase, $\bar{A}$ and $R$ track $P$ until it goes past 85 . With population effects when $P$ reaches the range [15,85], $R$ no longer increases but remains fixed at $\approx 15$ until $P \approx 65$. As $P$ increases, the sum of the population effects decreases in (d). 


\section{The Regulatory Mechanism}

Initial intuitions would suggest that as the external perturbations, $P$, increases, the population would respond to this selection pressure with the mean optimal resource level, $\bar{A}$, rapidly converging to the current resource level and tracking it as $P$ increases. The regulation evidenced when the population exerts an effect on the resource can be shown with the following: Initially the resource, $R$, begins below the viable range of any individual. As $P$ increases, $R$ will reach 15 and so there will be an individual that has non-zero fitness. We call this individual $A_{\text {best }}$. Given a random initial population, it is equally likely to have an $e$ or $E$ allele. If it has an $e$ allele, then as it increases in frequency, the effect the population has on the resource, $\sum_{i} \Theta_{i}$, becomes negative and so the rate of increase of $R$ decreases. Further increases in $P$ lead to further decreases in $\sum_{i} \Theta_{i}$ with $R$ remaining at the same value. Regulation is achieved via this simple negative feedback process.

Regulation may also be achieved if $A_{\text {best }}$ has the $R$ increasing $E$ allele. As $A_{\text {best }}$ increases in the population, which it will do as it has the highest fitness of any individual, $\sum_{i} \Theta_{i}$ will increase and $R$ will move past the optimal resource value of $A_{\text {best }}$. As $R$ moves away from this individual's optimum resource value, it will move towards the nearest individual with a higher $A$ allele and so a new $A_{\text {best }}$ is established. Once again this individual will have an $e$ or $E$ allele with equal probability. If it too has the increasing $E$ allele then $R$ will continue to increase, transit this $A_{\text {best }}$ and move nearer towards the individual with the nearest highest $A$ allele. Given a 0.5 probability of $R$ moving towards an individual with an $e$ allele, regulation on average will be rapidly established as an individual with a reducing $R$ allele will soon be encountered. As this increases in frequency a stable resource value, $R *$, will be established between the $\left[E, A_{\text {low }}\right]$ type and the $\left[e, A_{h i g h}\right]$ type. Allowing for stochastic reproduction and death, this produces two dominant sub-populations that straddle the resource variable and force it in opposing directions. See Figure 2. If $P$ were to increase, $R$ would increase and move closer to the optimum of the $\left[e, A_{h i g h}\right]$ type, this would increase the fitness of this type, lead to a decrease of $\sum_{i} \Theta_{i}$ and so reduce the increase in $R$. If $P$ were to decrease, the fitness of the $\left[E, A_{\text {low }}\right]$ type would increase and so $R$ would increase. This results in $R$ remaining fixed for varying $P$ and thus is equivalent in this respect to the proportional integral controller elements of detector, regulator and effector which seek to maintain a zero steady state error for the variable.

The mechanism of regulation in the model can be understood as an example of a rein control system. Rein control was introduced by Clynes [14] where it is hypothesised that certain variables (e.g. core body temperature in mammals) are maintained within a range of values by separate, unidirectional control reins that oppose forces that seek to perturb the variable. The rein control concept has since been developed in a physiological context [15] and latterly applied to analysis of a simplified Daisyworld model [16][17][18]. In this model the $\left[E, A_{\text {low }}\right]$ and the $\left[e, A_{h i g h}\right]$ sub-populations can be regarded as unidirectional control reins. Varying the strength of these reins (changing the numbers of individuals within 
each sub-population) leads to the resource being regulated to within a narrow range over varying external perturbations.

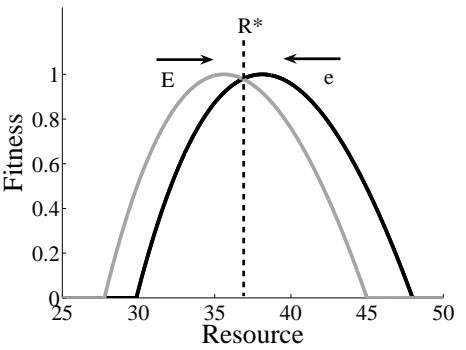

(a)

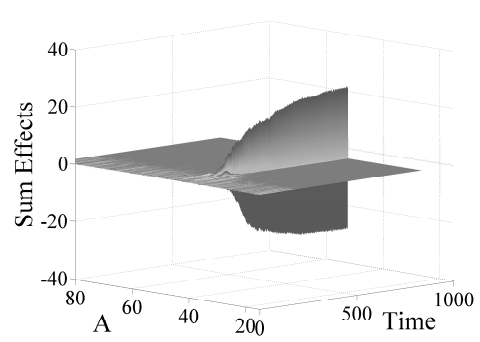

(b)

Fig. 2. In (a) the $\left[A_{\text {low }}, E\right]$ type's fitness range is plotted with a solid grey line, the $\left[A_{h i g h}, e\right]$ type's fitness range is plotted with a solid black line. The vertical dashed black line marks the value for $R *$. The resource will be maintained around this value. If the the $e$ allele were to increases in frequency, due to an increase in $R$ (produced by internal or external perturbations) the change in the sum of effects leads to a counter opposing force acting to reduce this increases. Similarly if the $E$ allele were to increase in frequency, due to an decrease in $R$ the sum of effects leads to a counter opposing force acting to reduce this decreases. The emergence of a regulatory epoch is shown in (b). Individuals are collated into 70 distinct $A$ trait bins which are plotted on the bottom left axis. For each binned $A$ trait, the sum of population effects is plotted on the vertical axis. $P$ is fixed at 37 for 1000 time steps. The initially diverse population, rapidly converges to two sub-populations: $\left[A_{l o w}, E\right]$ and $\left[A_{h i g h}, e\right]$ that produce an equal increasing and decreasing effect on $R$ with the net result that $R$ remains at this value.

\section{Results}

In order to assess the effects of diversity in the model ecosystem, simulations with varying mutation rates, $\mu$, were performed. Higher values for $\mu$ produce more diverse populations as mutation counter-acts the selective force that would have the entire population converge to the regulating sub-populations. As this random force would upset the regulatory balance in the population, it would be initially expected that the higher the value for $\mu$ and so the greater the diversity in the population, the lower the model's stability. This proves not necessarily to be the case.

100 simulations were performed for the 11 mutation rates over the range $[0,1]$. For each simulation, the total amount of time that $R$ was regulated along with the ecosystem's biodiversity was recorded. Results are plotted in Figure 3. Biodiversity was measured in terms of the number of unique $\mathrm{A}$ alleles in the population. The A allele for each individual was rounded to the nearest integer 
and a sum of present A alleles recorded (the maximum being 71) at every time step. This total was then divided by the number of time steps over a simulation. $R$ was judged to have been regulated if the average rate of increase of $R$ was less than $P$ over 200 time steps. It was necessary to average $d R / d t$ in this manner as the stochastic GA produced a certain amount of noise in $R$. Rather than decrease, regulation increases with increasing mutation until $\mu=0.3$. Increasing $\mu$ past 0.4 results in a sharp decrease in regulation.

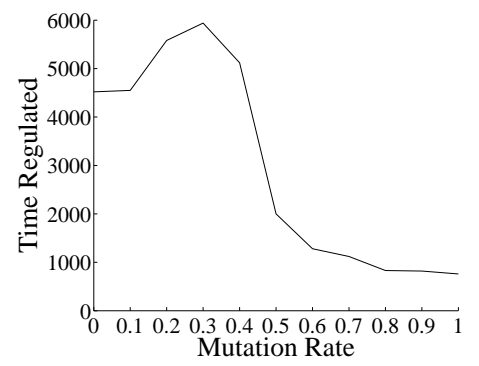

(a)

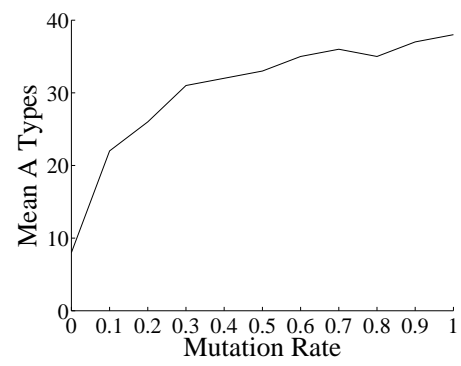

(b)

Fig. 3. 100 simulations of 100,000 time steps were performed for each mutation rate. For each simulation, the length of time that the resource was regulated was recorded $(d R / d t<d P / d t$ over 200 time steps). The mean number of turns that $R$ was regulated for each mutation rate is plotted on the vertical axis in (a). Regulation increases as mutation rates increase from 0 until an optimum of 0.3 . Mutation rates higher than 0.4 lead to sharply reduced regulation. In (b) the mean amount of diversity (as measured with unique A types) over 100 simulations for each mutation rate is plotted on the vertical axis. Increasing mutation, increases diversity with the greatest rate of increase over the range $[0,0.3]$

This relationship can be explained by the different effect mutation will have on the system's resistance and resilience. Higher $\mu$ will tend to decrease the system's resistance. As the mutation rate increases, the length of any particular regulatory epoch decreases as at the limits of regulation mutation will remove individuals from the regulating sub-population and so decrease the maximum effect the organisms are able to exert on the environment. Having a more diverse population can increase the system's resilience however. Higher mutation rates increase the number of individuals outside of any current regulatory sub-population. These 'outliers' can prove to be the seed of a new regulatory epoch and so allow the system to recover regulation after the collapse of a previous regulatory epoch. See Figure 4. For a range of mutation rates, the increase in resilience is greater than the decrease in resistance and so total regulation increases. In that respect a more diverse system can prove to be more stable. 


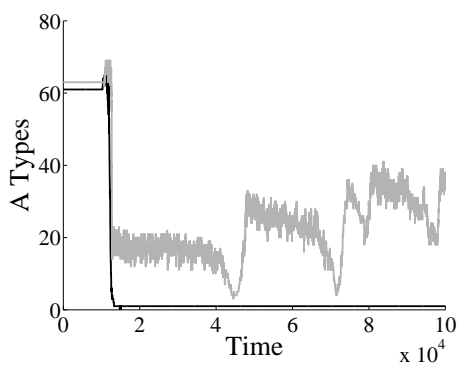

(a)

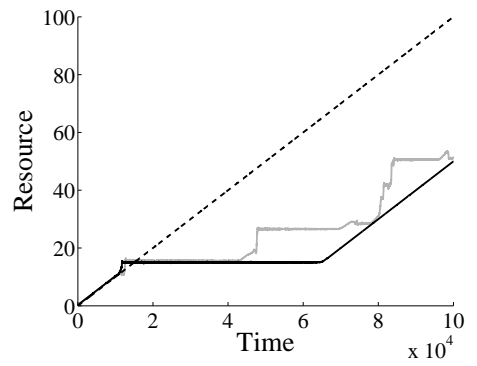

(b)

Fig. 4. Results of two simulations are plotted: $\mu=0$ with solid black line; $\mu=0.3$ solid grey line. In (a) the number of unique integer value A types in the population is plotted (the A allele for each individual was rounded to the nearest integer). With no mutation diversity rapidly decreases to 2 with the establishment of a regulatory epoch. With mutation the population rapidly decreases to approximately 20 , and then undergoes a series of decreases and sharp increases as new regulatory epochs are established. In (b) $P$ is plotted with a dashed black line, $R$ is plotted with solid lines. The regulatory epoch with no mutation is longer than that with 0.3 mutation. However total regulation is greater with 0.3 mutation as subsequent regulatory epochs are established. The establishment of these new regulatory epochs is shown in the sharp spikes in diversity in (a). The sharp transitions and multiple regulatory epochs observed are similar to the Daisyworld-type regulating model of [19].

\section{Conclusion}

A simple model ecosystem has been presented. As well as responding to changes in the environment, the organisms also exerted an effect on the environment. This led to the regulation of an environmental resource over a range of external perturbations. Increasing the mutation rate increased the diversity of the population by seeding individuals outside of the regulating sub-populations. It was found that higher mutation rates decrease the system's resistance in that the length of any regulatory epoch tends to be reduced. However higher mutation rates increase the system's resilience as increased diversity increases the probability that new regulatory epochs can be established. For a range of mutation rates, the increase in resilience was greater than the decrease in resistance and so produced an increase in the total amount of time that the ecosystem was able to regulate against the external perturbation. These results support the conclusions of [11] in particular the observation that in order for an ecosystem (a multi-species Daisyworld) to be able to respond to external perturbations there must be sufficient biodiversity. In this model, whilst biodiversity has no 'value' for the maintenance of a particular stable state, it can prove essential to the establishment of new stable states and as such biodiversity may be an important measure of the overall robustness of an ecosystem. This suggests that decreasing the complexity of an ecosystem by decreasing its biodiversity may 
imperil the entire ecosystem and not just those individual species threatened with extinction.

\section{References}

1. Odum, E. P.: Fundamentals of Ecology. Saunders, Philadelphia, (1953).

2. Elton, C. S.: Ecology of Invasion by Animals and Plants. Chapman \& Hall, (1958).

3. May, R. M.: Will a large complex system be stable? Nature 238 (1972) pp 413-414.

4. Gardner, M. A., Ashby, W. R: Connectance of large dynamic (cybernetic) systems: critical values for stability. Nature (1970) pp 228-784.

5. McCann, K. S: The diversity-stability debate. Nature 405 (2000) pp 228-233.

6. McDonald-Gibson, J.: Investigating Gaia: A new mechanism for regulation. Thesis for degree of Masters of Science of Evolutionary and Adaptive Systems, University of Sussex (2006).

7. Watson, A., Lovelock, J: Biological Homeostasis of the global environment - the parable of Daisyworld. Tellus B 35 (1982) pp 284-289.

8. Laland, K. N., Odling-Smee, J., Feldman, M.W.: The evolutionary consequences of niche construction: a theoretical investigation using two-locus theory. Journal of Evolutionary Biology 9 (1996) pp 293-316.

9. Laland, K. N., Sterelny, K.: Perspective: seven reasons (not) to neglect niche construction. Evolution 60, (2006) pp1751-1762

10. Wood, J., Ackland, G. Dyke, J. Lenton, T., William, H.: Daisyworld: A review. Reviews of Geophysics. Forthcoming.

11. Lovelock, J.: A numerical model for biodiversity. Phil. Trans. R. Soc. 338 (1992) pp 383-391.

12. Harding, S.: Food web complexity enhances community stability and climate regulation in a geophysical model. Tellus B 51 (1999) pp 815-829.

13. Harding, S., Lovelock, J.: Exploiter mediated coexistence and frequency dependent selection in a numerical model of biodiversity. J. Theor. Biol. 182 (1996) pp 109-116.

14. Clynes, M.: Cybernetic implications of rein control in perceptual and conceptual organization. Ann. NY Acad. Sci. 156 (1969) pp 629-670

15. Saunders, P.T., Koeslag, J., Wessels, A..: Integral Rein Control in Physiology. Journal Theoretical Biology, 194 (1998) pp 164-173.

16. Harvey, I.: Homeostasis and Rein Control: From Daisyworld to Active Perception. Proceedings of the Ninth International Conference on the Simulation and Synthesis of Living Systems, ALIFE'9, Pollack, J., Bedau, M,. Husbands, P., Ikegami, T., and Watson, R.A. (eds), (2004) pp 309-314.

17. Dyke J., Harvey I.: Hysteresis and the Limits of Homeostasis: from Daisyworld to Phototaxis. Proceedings of VIIIth European Conference on Artificial Life, ECAL 2005, Capcarrere, M., Freitas, A., Bentley, J., Johnson, C. and Timmis, J. (eds). Springer-Verlag. (2005) pp 332-342.

18. Dyke, J., Harvey, I.: Pushing up the daisies. Artificial Life X, Proceedings of the Tenth International Conference on the Simulation and Synthesis of Living Systems Proceedings Editors: L.M. Rocha, L.S. Yager, M.A. Bedau, D. Floreano, R.L. Goldstone, A. Vespignani, MIT Press. (2006) pp 426-431.

19. William, H., Noble, J.: Evolution and the Regulation of Environmental Variables. Proceedings of VIIIth European Conference on Artificial Life, ECAL 2005, Capcarrere, M., Freitas, A., Bentley, J., Johnson, C. and Timmis, J. (eds). Springer-Verlag. (2005) pp 332-342. 